\title{
First bilateral simultaneous implantation with fully implantable middle ear hearing device
}

\author{
Magdalena Lachowska ${ }^{1}$, Kazimierz Niemczyk ${ }^{1}$, Alain Yazbeck², Krzysztof Morawski ${ }^{1}$, \\ Antoni Bruzgielewicz ${ }^{1}$
}

1Department of Otolaryngology, Medical University of Warsaw, Poland

2Otologics LLC, Boulder, Colorado, USA

Submitted: 19 August 2010

Accepted: 13 March 2011

Arch Med Sci 2012; 8, 4: 736-742

DOI: 10.5114/aoms.2012.30299

Copyright @ 2012 Termedia \& Banach

The majority of moderate to severe sensorineural hearing loss individuals must rely on amplification of the acoustic signal to improve communication skills. This aim is generally accomplished with conventional air conduction hearing aids [1]. In some cases, hearing aid wearers report difficulties and limited adoption of hearing aids due to ear canal occlusion, pain or irritation of the skin in the ear canal, social stigma, and cosmetic issues [2]. The fully implantable middle ear hearing device is now an alternative solution for those patients. It resides completely underneath the skin behind the ear, with nothing in the external ear canal. The Otologics Carina implant is composed of an electronic capsule, microphone, and electromagnetic transducer. The battery, magnet, digital signal processor, coil, and connector make up the electronic capsule [1, 2]. The sensitive subcutaneous microphone picks up the acoustic signal, which is then translated into a digital signal, amplified according to the wearer's needs, and converted into the electrical signal. This electrical signal is sent to the electromagnetic transducer, which translates it into a mechanical motion that directly stimulates the ossicles. The tip of the transducer touches the middle ear ossicular chain, round or oval window and in this way bypasses the external ear canal [1-5].

Besides the fully implantable part, three other components are also important for this hearing device: the programming system, the charger, and the remote control. The charging time of the battery in the newest Carina device typically requires $45 \mathrm{~min}$ to $1 \mathrm{~h}$, if performed daily.

The fully implantable middle ear hearing system Carina is designed to deal with moderate to severe hearing loss in adults with normal middle ears, and no signs or symptoms of retrocochlear, central auditory or functional components. Most recently it has been successfully used in patients with ossicular chain malformations in conductive and mixed hearing loss [6-9].

In this paper the authors present a case report of the first bilateral simultaneous implantation with a fully implantable middle ear hearing device. Candidacy criteria and preoperative and postoperative audiological results are reported, and advantages obtained with these implants are discussed.

One patient was included in this retrospective case report. To our best knowledge it is the first reported case of bilateral simultaneous implantation with a fully implantable middle ear hearing device. The surgery took place in the Department of Otolaryngology at the Medical University of

\author{
Corresponding author: \\ Magdalena Lachowska \\ MD, PhD \\ Department \\ of Otolaryngology \\ Medical University of Warsaw \\ 1a Banacha \\ 02-097 Warsaw, Poland \\ Phone: +48 225992521 \\ Fax: +48 225992523 \\ E-mail: \\ mlachowska@wum.edu.pl
}


Warsaw in Poland. The subject was a Polish speaking female, 18 years old at the time of the surgery. She suffered from moderate bilateral sensorineural hearing loss and was using conventional digital air conduction hearing aids, which she found to be very stigmatizing and embarrassing, even though they were in-the-ear (ITE) hearing aids. The patient had no signs or symptoms of middle or inner ear malformations, no retrocochlear, central auditory or functional components, and no medical contraindications to surgery or middle ear implantation. She was fully informed about the Otologics Carina device and all the details associated with the bilateral surgery and its possible associated risks, as well as about aural rehabilitation after the implantation, possible complications and auditory outcomes published in the world literature so far. Sequential unilateral versus simultaneous bilateral surgery risks were discussed with the patient. Her expectations of device benefit were realistic.

The surgery was performed by a very experienced otosurgeon on July 7, 2009. The leading surgeon was Professor Kazimierz Niemczyk MD, PhD, the Head of the Department of Otolaryngology at the Medical University of Warsaw in Poland. The surgical technique was similar to one already described in the literature by Jenkins et al. [1, 2], and was the same on both ears. Bilateral implantation of Carina devices took place during the same surgical session.

The surgery was begun on the right side. An incision, similar to that for cochlear implantation, was created posteriorly to the right auricula. A cutaneous-muscular flap was developed and the mastoid and retroauricular regions were visualized. A small superior atticotomy was created and the head of the malleus and the body of the incus were exposed. The mounting bracket with the transducer of the device was introduced. The mounting bracket was fitted to the mastoid bone using screws to secure the position of the transducer (Figure 1).

Bone beds for the microphone and the capsule were drilled so that they could be properly positioned and secured. The microphone was placed in the retroauricular region on the mastoid. The tip of the transducer was advanced and safely placed directly on the incus. The positioning of the tip was evaluated using the special software Transducer Loading Assistant (TLA) developed by Otologics. The TLA is used to ensure correct and safe placement of the transducer and to improve the coupling efficiency along with the ease and confidence for the surgeon [4]. The integrity of the system was checked intraoperatively. Finally the wound on that side was closed and wound dressing applied. Next, the head of the patient was turned about $90^{\circ}$ to the other side with special care taken in order to secure the first operated ear. The surgery of the sec- ond ear (left) was performed with the same steps as the first ear. For the first part of the surgery (right ear) monopolar electrocoagulation was used to ensure hemostasis. For the second part of the surgery (left ear) bipolar electrocoagulation was used in order to not interfere with the Carina system already implanted on the other side.

The postoperative healing was uneventful, with no further complications.

Ten weeks postoperatively, the middle ear implants were activated using the OtoFit fitting software. Further adjustments of the internal audioprocessor along with feedback reduction, and audiological tests took place 1,2 , and 7 months later $(3,4$, and 9 months after the surgery). Every fitting session was preceded and then followed by audiological tests. Additional audiological tests were performed 3 months after activation of the implants (5 months after the surgery).

Air and bone conduction monaural thresholds using headphones and bone conductor were obtained pre- and postoperatively. The last preoperative measurements were performed one day prior to surgery. Binaural pure tone thresholds in free field, as well as speech discrimination scores were tested postoperatively. Free field measurements were performed in both unaided and aided conditions. The position of the patient was $1 \mathrm{~m}$ away from the loudspeakers at 0 degrees azimuth; patient was directly facing the speakers at all times during testing. For aided conditions, the patient was asked to set the volume control in both implants at the most comfortable listening level based on speech input at $65 \mathrm{~dB}$ sound pressure level (SPL). The Polish monosyllabic discrimination test for speech audiometry was used; word lists were selected randomly to avoid learning effects, and at a series of increasing levels that varied in $10 \mathrm{~dB}$ steps. This speech testing protocol mapped out the speech discrimination performance from $0 \%$ to

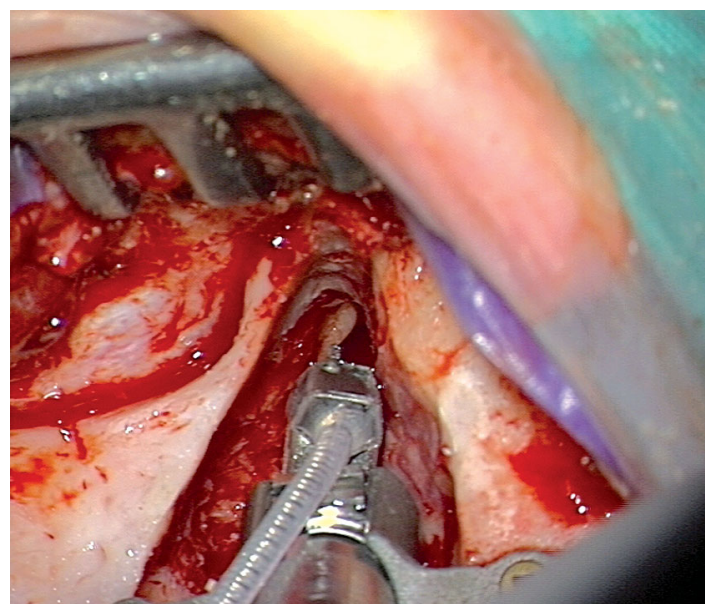

Figure 1. Intraoperative view of the mounting bracket and the transducer of the fully implantable middle ear implant Carina placed on the ear 
maximum discrimination obtained by the patient; in this case it was 100\%. Pure tone averages (mean pure tone thresholds) were derived by averaging the preoperative thresholds at 500, 1000, 2000 and $4000 \mathrm{~Hz}$. All audiological tests were performed in the attenuated sound room with specially designed walls in the Auditory Laboratory of the Department of Otolaryngology at the Medical University of Warsaw. The room itself and its walls were designed in a way to prevent resonance of the sound waves used for audiological tests. The walls of the room are made of special absorbent material. Due to these features of the room, we do not deal with a negative effect of standing waves and we are able to perform free field audiometry using pure tones.

In every fitting session, the patient was asked about: 1) the battery charging time, 2) daily use of the implants, as well as 3) her comfort and freedom in wearing them (ear occlusion effect, showering, swimming pool activities, freedom in other sport activities, etc). These are standard questions used in cases of hearing impaired patients wearing hearing devices in the Department of Otolaryngology. In cases of fully implantable middle ear implants additional questions are asked in regard of perception of patients' body noises (including noises from clothes) and their own voice.

The difference between postoperative (1, 2 and 3 months after the activation of middle ear implants, which is 3, 4, and 5 months after the surgery respectively) and preoperative pure tone thresholds were clinically not significant and are illustrated in Figure 2.

A Pure tone audiometry bone conduction right ear $[\mathrm{Hz}]$

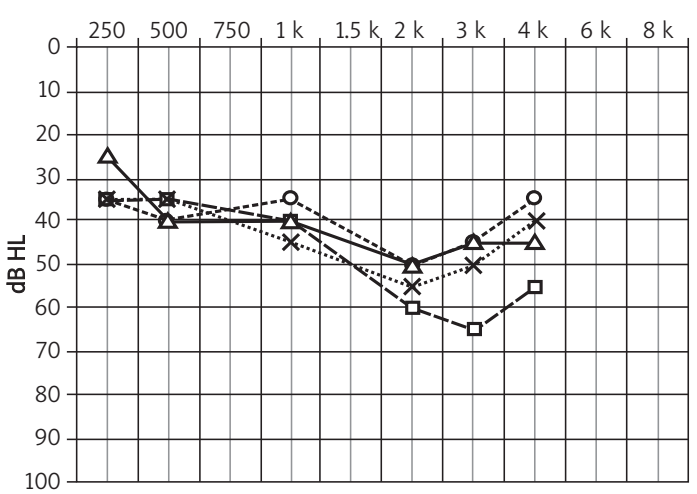
Pure tone audiometry bone conduction left ear $[\mathrm{Hz}]$

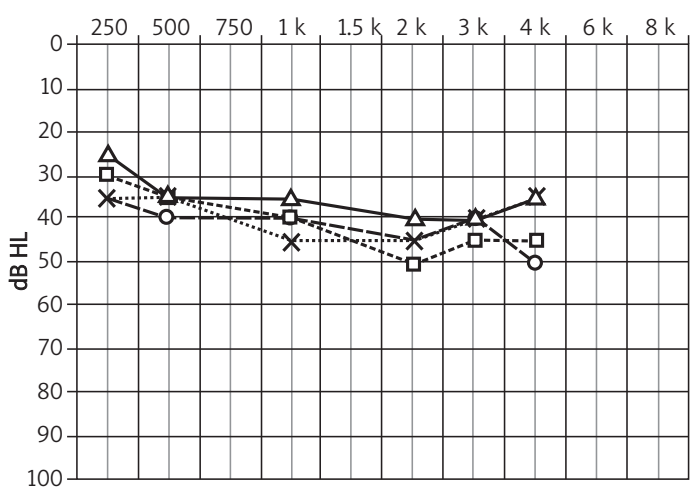

$$
\begin{aligned}
& \text {-o- BC pre-op Jul } 2009 \\
& \text {...... BC post-op Sep } 2009 \\
& \triangle-\text { BC post-op Dec } 2009 \\
& \text {--ם-- BC post-op April } 2010
\end{aligned}
$$

B

Pure tone audiometry air conduction right ear $[\mathrm{Hz}]$

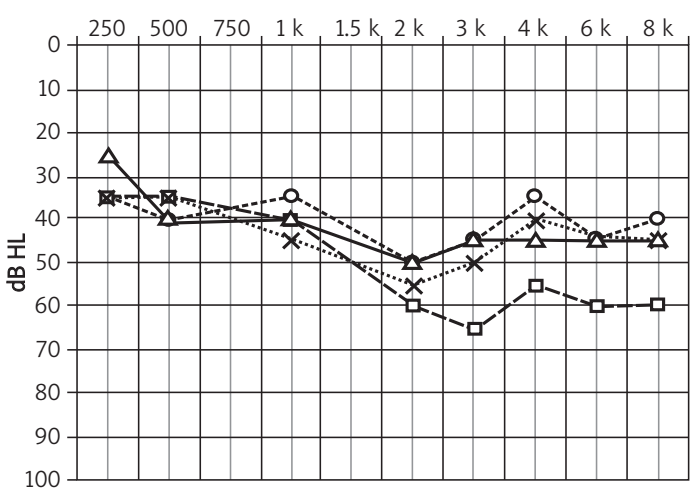

Pure tone audiometry air conduction left ear $[\mathrm{Hz}]$

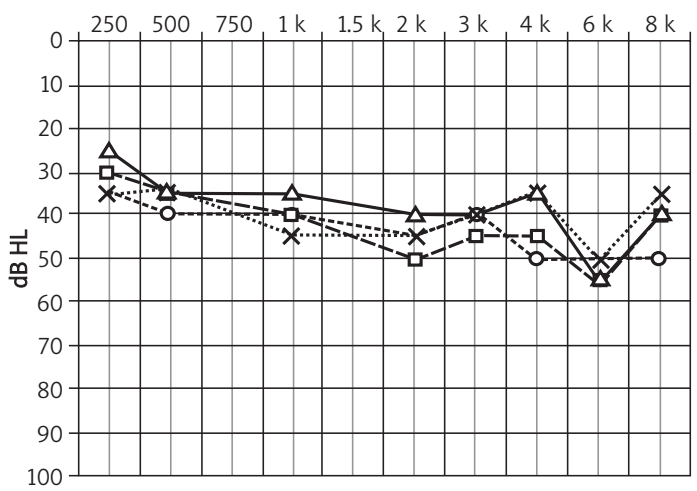

$$
\begin{aligned}
& \text {-o- AC pre-op Jul } 2009 \\
& \text { …-. AC post-op Sep } 2009 \\
& -\triangle \text { - AC post-op Dec } 2009 \\
& \text {--ם-- AC post-op April } 2010
\end{aligned}
$$

Figure 2. Preoperative (pre-op) and postoperative (post-op) pure tone thresholds (in $\mathrm{db} \mathrm{HL}$ ) for: $\mathrm{A}$ ) bone conduction (BC) and B) air conduction (AC), with both fully implantable middle ear hearing devices switched off 
A change was noted 7 months after activation of the implant in the right ear (9 months after the surgery): worsening of the patient's hearing on the right side. This change was also noted by the patient a few weeks before that fitting session and is not associated with the surgical procedure, taking into account the elapsed time between the date of implantation and the reported drop in hearing sensitivity. The mean pure tone aided threshold in free field (with both implants turned on) was 36.25 $\mathrm{dB}$ HL just after activation (first fitting). Similar mean aided thresholds were obtained 1 month later at the second fitting session. Seven months after activation of both implants (fourth fitting session, 9 months after the surgery), aided free field pure tone audiometry thresholds were at the mean level of $32.5 \mathrm{~dB} \mathrm{HL}$; the unaided condition was at $40 \mathrm{~dB} \mathrm{HL}$ (Figure 3).

This result shows an average functional gain of $7.5 \mathrm{~dB}$. The functional gain may be calculated by determining the difference between the aided and unaided postoperative pure tone thresholds in free field audiometry. The bilateral aided results for $4000 \mathrm{~Hz}$ showed no functional gain due to the feedback problems at that frequency; the gain in this range had to be decreased to avoid feedback (Figure 4).

The bilateral aided results for $500 \mathrm{~Hz}, 1000 \mathrm{~Hz}$ and $2000 \mathrm{~Hz}$ (Figure 3) show functional gains of $5 \mathrm{~dB}, 10 \mathrm{~dB}$, and $20 \mathrm{~dB}$ respectively. It is worth mentioning that the best aided threshold results that can be obtained for the frequency range from $500 \mathrm{~Hz}$ to $6000 \mathrm{~Hz}$ (it is the frequency range accessible for gain changes in this device) with Carina implants are
25-30 dB HL. As shown in Figure 3, the aided thresholds for $500 \mathrm{~Hz}, 1000 \mathrm{~Hz}, 2000 \mathrm{~Hz}$, and $3000 \mathrm{~Hz}$ were $30 \mathrm{~dB} \mathrm{HL}, 25 \mathrm{~dB} \mathrm{HL}, 30 \mathrm{~dB} \mathrm{HL}$, and $30 \mathrm{~dB}$ HL respectively. Figure 3 also presents aided and unaided pure tone thresholds measured for each ear separately. During aided measurements for each ear separately, one ear was aided with the implant while the opposite ear had its device switched off in addition to being plugged with a blinded insert.

As mentioned before, the gain at frequency of $4000 \mathrm{~Hz}$ had to be decreased in order to reduce feedback. Subjectively, the patient also reported an additional feedback problem in the left ear. This problem was successfully resolved by decreasing the gain at frequency of $3000 \mathrm{~Hz}$ in the left Carina device, resulting in no functional gain in the left ear at that frequency.

Before the surgery the $100 \%$ speech discrimination performance for the right and the left ear separately was obtained at a stimulation level of $85 \mathrm{~dB}$ SPL. Four weeks after activation, the maximum aided discrimination score of $100 \%$ was recorded at a presentation level of $55 \mathrm{~dB}$ SPL (both ears aided condition). Seven months after activation with both implants switched on (both ears aided condition) the $100 \%$ discrimination score threshold was $40 \mathrm{~dB}$ SPL versus $60 \mathrm{~dB} S P L$ in the unaided conditions. These results show a speech discrimination functional gain of $+20 \mathrm{~dB}$. Figure 5 presents unaided and aided speech discrimination performance for both ears and each ear separately, 7 months after activation of the implants.

With regards to postoperative adverse effects in our patient, no extrusion of the device, loss of exter-
A Free field pure tone thresholds right and left ear separately

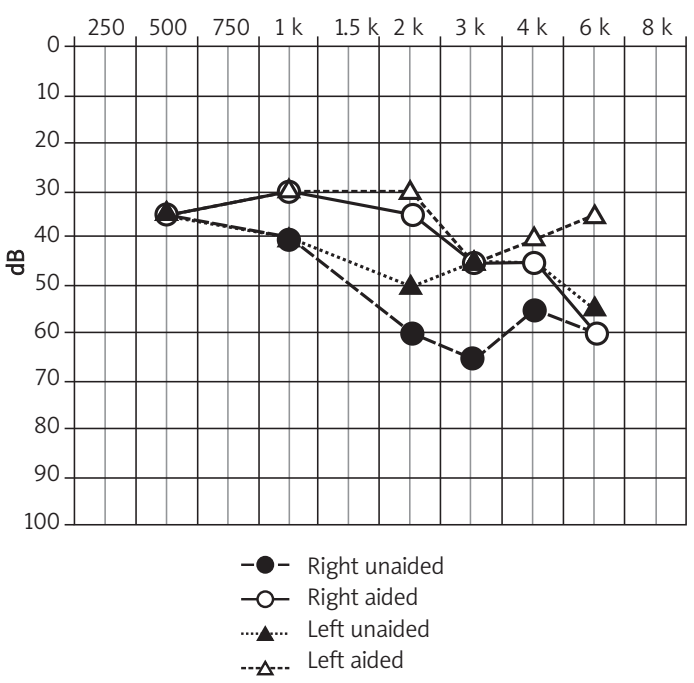

Free field pure tone thresholds both ears

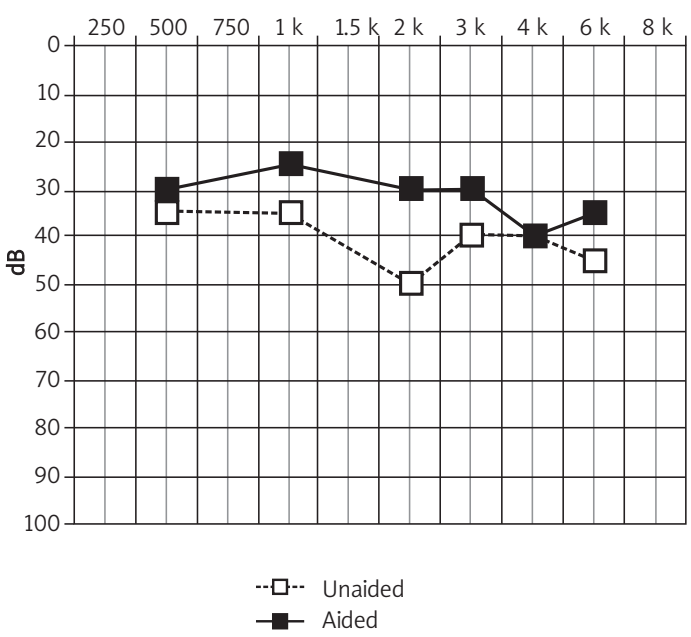

Figure 3. Aided and unaided free field pure tone thresholds (in $\mathrm{dB} \mathrm{HL}$ ) measured for: A) each ear separately, and B) for both ears 7 months after activation of the fully implantable middle ear hearing devices 
A

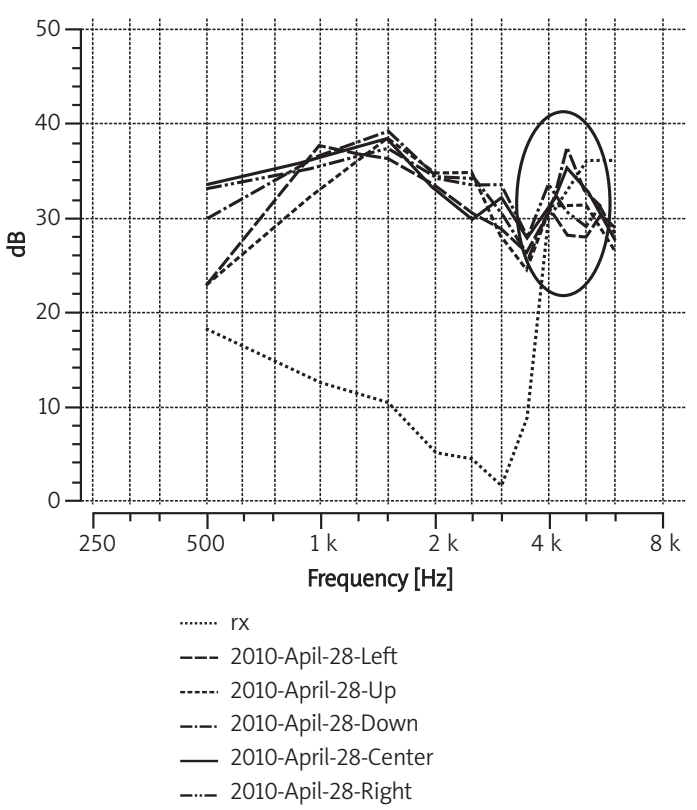

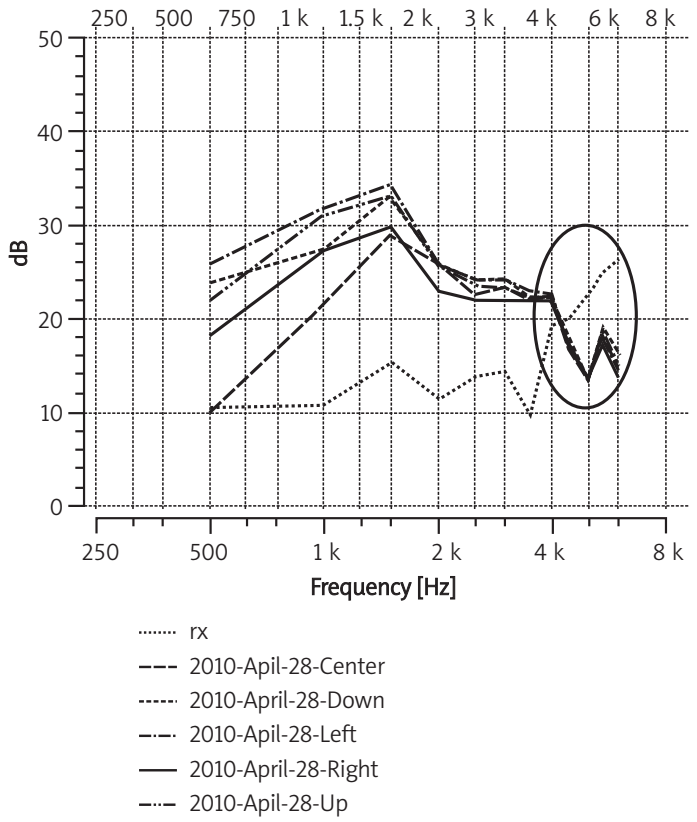

Figure 4. Screen shots from the fitting session performed 7 months after activation of the fully implantable middle ear hearing devices - positional noise measurements for: $\mathrm{A}$ ) the left ear, and $\mathrm{B}$ ) the right ear. The circles mark the region of feedback problems; the gain in that frequency range (about $4000-6000 \mathrm{~Hz}$ ) had to be decreased

A

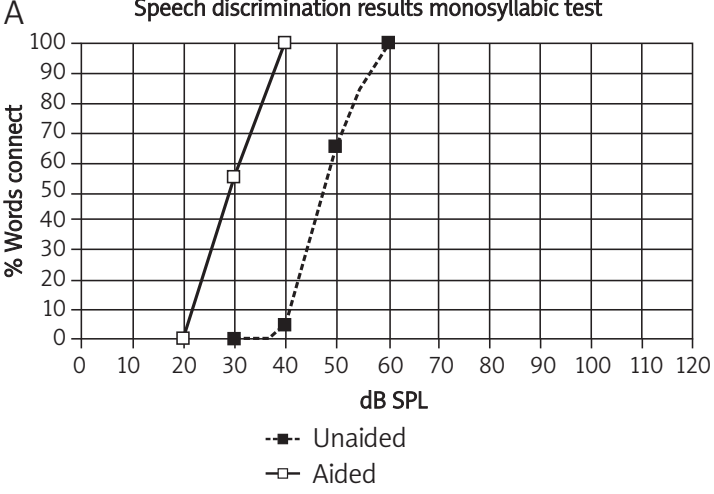

B Speech discrimination results monosyllabic test

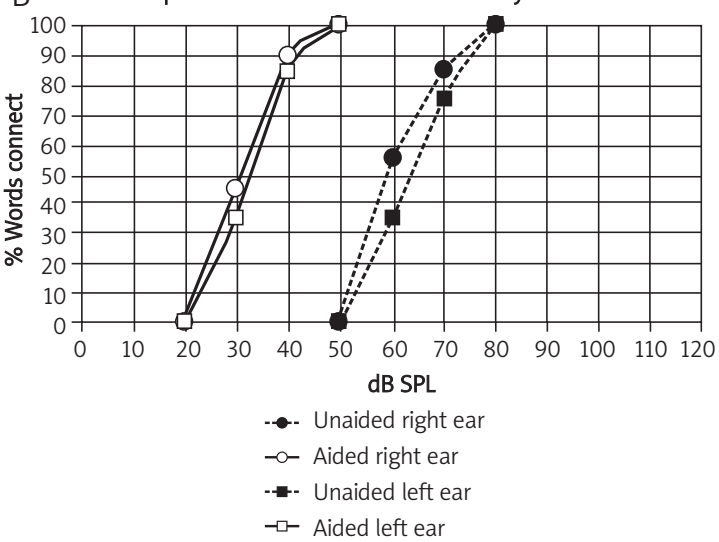

Figure 5. Aided to unaided free field speech discrimination tests (dB SPL) for: A) both ears, and B) each ear separately, 7 months following activation of the fully implantable middle ear hearing devices

nal communication, device failure or increased charging time were observed.

The freedom and the cosmetic advantages obtained with the fully implantable middle ear hearing devices are greatly appreciated by the patient. She also profits from using it in all environments with no limitations of normal activities. The charging time is about $1 \mathrm{~h}$ for both implants and is done on a daily basis. She uses both implants all day long, switching them off only when she goes to sleep. Because the patient uses both implants at the same time, she reports subjective benefits from bilateral stimulation. At initial activation, the patient perceived her body and clothes noises, and her own voice as loud or different. Those subjective problems systematically decreased with time required for adaptation to the implanted middle ear devices and following fitting sessions.

The results presented in this case report of the first bilateral simultaneous implantation with a fully implantable middle ear hearing device provide several initial insights regarding preoperative and postoperative audiological data and advantages obtained with bilateral implants. The surgery itself presented no unusual challenges or difficulties for the surgeon, except that it was performed bilater- 
ally and the implantation of two devices took place during the same surgical session. To our best knowledge, it is the first reported case of such implantation. Special care must be taken during transducer placement on the ossicular chain in order not to disarticulate the chain or damage the cochlea. Intraoperative testing with Transducer Loading Assistant software was of great help here [4]. The surgery is described as safe for residual hearing by most authors $[1,2,9]$. All the surgery risks associated with one (bilateral simultaneous) versus two (unilateral sequential) operations along with anesthesia risks for one versus two were taken into consideration for decision making. Another important factor was the leading surgeon's great expertise in otosurgery. It was decided, with the patient's fully informed and signed consent, that the surgery would be performed in both ears during one surgical session instead of a prolonged sequential procedure which would require two operations. In our case the bilateral implantation with middle ear fully implantable hearing devices did not result in a clinically important change of the patient's cochlear status, as demonstrated by air and bone conduction thresholds, which were similar to the preoperative measurements.

The presented results in this case report are in agreement with those reported in the literature, and confirm that the fully implantable middle ear implant Carina is a viable alternative treatment for moderate sensorineural hearing loss [1, 2, 9]. The difference to other authors is that the bilateral implantation in the presented patient was performed simultaneously in one surgical session. The aided versus unaided pure tone thresholds and speech discrimination test results show the patient's benefits.

Jenkins et al. [2] reported some complications with the Carina device that occurred during the 12-month data collection, which included partial device extrusion, loss of external communication, and increased charging time beyond $1.5 \mathrm{~h}$. In contrast, Bruschini et al. [9] did not report any of the complications mentioned above. In our study the data collection time was shorter (follow-up time was 7 months after the implants' activation, which was 9 months after the surgery), but our patient also has not experienced any of those problems so far. The learning experience and the evolution in the design of the device have helped to reduce to a minimum the adverse effects experienced in the very early stages of this technology.

Subjective benefits reported by patients are taken into account by many authors [2, 9]. The fully implantable middle ear hearing device system offers the same freedom and comfort as the natural auditory system; it can be used in situations that are not suitable for conventional air conduction hearing aids.
The freedom and cosmetic advantages obtained with the fully implantable devices are greatly appreciated by our patient too. There is no visible evidence that the hearing prostheses are being worn. The patient reports subjective good sound location and feels safe in everyday life. The patient stated that simultaneous implantation offered her aural rehabilitation with both ears aided conditions from the beginning, so she did not have to start with one middle ear implant rehabilitation while waiting for the second to be implanted.

At the initial activation of Carina devices, the patient complained about her body and clothes noises and her own voice as being loud or different. This feature was expected because conventional hearing aids do not amplify transmitted sounds from the body. After acclimatization to the bilateral fully implantable middle ear implants the patient reported those sounds to be at a relatively normal level for body sounds including her voice and clothes. This subjective statement seems to support the manufacturer's claims of a relatively normal body sound level. The signal processing feature included in the system is designed to present them in the output as comparable to that perceived by persons with normal hearing.

The presented results of one patient suggest that the bilateral simultaneous implantation of the fully implantable middle ear hearing devices is an effective procedure. The use of only one implant would exclude the patient from binaural hearing, and the simultaneous bilateral implantation offers the opportunity to start aural rehabilitation with both ears in aided conditions from the beginning. This solution might be an alternative to binaural conventional hearing aids for selected patients with bilateral sensorineural hearing loss. This case report presents the results of 7-month post-activation follow-up (9-month postoperative follow-up) in 1 patient. In our opinion the longer use of bilateral middle ear implants and aural rehabilitation along with optimization for gain and speech understanding should improve the patient's results. This statement is based on improvement of the patient's performance in time following activation of both devices. Further clinical studies are required to confirm these results in a larger group of patients, define inclusion criteria for bilateral simultaneous implantation including safety concerns, and draw more general conclusions.

\section{Acknowledgments}

The authors would like to thank technicians from the Auditory Laboratory of the Department of Otolaryngology at the Medical University of Warsaw (Hanna Siewko, Beata Klukowska, and Magdalena Baniewicz) who performed audiological tests included in this study. 
The authors report a declaration of interest: one of the co-authors of the submitted manuscript (Mr. Alain Yazbeck) is an employee of Otologics LLC Boulder (Colorado, USA). Otologics Carina (the fully implantable middle ear hearing device described in this article) is a trademark of Otologics, LLC.

\section{References}

1. Jenkins HA, Atkins JS, Horlbeck D, et al. US phase I preliminary results of use of the otologics MET fully implantable ossicular stimulator. Otolaryngol Head Neck Surg 2007; 137: 206-12.

2. Jenkins HA, Atkins JS, Horlbeck D, et al. Otologics fully implantable hearing system: phase I trial 1-year results. Otol Neurotol 2008; 29: 534-41.

3. Jenkins HA, Pergola N, Kasic J. Anatomical vibrations that implantable microphones must overcome. Otol Neurotol 2007; 28: 579-88.

4. Jenkins HA, Pergola N, Kasic J. Intraoperative ossicular lading with the otologics fully implantable hearing device. Acta Otolaryngol 2007; 127: 360-4.

5. Martin C, Deveze A, Richard C, et al. European results with totally implantable Carina placed on the round window: 2-year follow-up. Otol Neurotol 2009; 30: 1196-203.

6. Siegert R, Mattheis S, Kasic J. Fully implantable hearing aids in patients with congenital auricular atresia. Laryngoscope 2007; 117: 336-40.

7. Tringali S, Pergola N, Ferber-Viart C, Truy E, Berger P, Dubreuil C. Fully implantable hearing device as a new treatment of conductive hearing loss in Franceschetti syndrome. Int J Pediatr Otorhinolaryngol 2008; 72: 513-7.

8. Lefebvre PP, Martin C, Dubreuil C, et al. A pilot study of the safety and performance of the otologics fully implantable hearing device: transducing sounds via the round window membrane to the inner ear. Audiol Neurotol 2009; 14: 172-80.

9. Bruschini L, Forli F, Santoro A, Bruschini P, Berrettini S. Fully implantable Otologics MET Carina device for the treatment of sensorineural heating loss Preliminary surgical and clinical results. Acta Otorhinolaryngol Ital 2009; 29: 79-85 